\title{
Social Class Predicts Emotion Perception and Perspective-Taking Performance in
}

\section{Adults}

\author{
Pia Dietze ${ }^{1}$ and Eric D. Knowles ${ }^{2}$ \\ ${ }^{1}$ Department of Psychology and Social Behavior, University of California, Irvine \\ ${ }^{2}$ Department of Psychology, New York University \\ [9947 words]
}

\begin{abstract}
Author Note
Pia Dietze https://orcid.org/0000-0002-3564-8961

Eric D. Knowles https://orcid.org/0000-0001-8525-1930

This research was supported by National Science Foundation grant \#1729295 to

E.D.K. The authors have no known conflicts of interest to disclose.

Correspondence concerning this article should be addressed to Pia Dietze, 5546

Social and Behavioral Sciences Gateway, University of California, Irvine, Irvine, CA 92617. Email: pdietze@uci.edu or pia.dietze@nyu.edu.
\end{abstract}




\begin{abstract}
“Theory of Mind" (ToM; people's ability to infer and use information about others' mental states) varies across cultures. In four studies $(N=881)$, including two preregistered replications, we show that social class predicts performance on ToM tasks. In Studies 1A and 1B, we provide new evidence for a relationship between social class and emotion perception: Higher-class individuals performed more poorly than their lower-class counterparts on the Reading the Mind in the Eyes Test, which has participants infer the emotional states of targets from images of their eyes. In Studies 2A and 2B, we provide the first evidence that social class predicts visual perspective-taking: Higher-class individuals made more errors than lower-class individuals in the Director Task, which requires participants to assume the visual perspective of another person. Potential mechanisms linking social class to performance in different ToM domains, as well as implications for deficiency-centered perspectives on low social class, are discussed. [150 words]
\end{abstract}

Keywords: Social Class, Culture, Theory of Mind, Director Task, Reading the Mind in the Eyes Test 


\section{Social Class Predicts Emotion Perception and Perspective-Taking Performance in Adults}

Theory of mind (ToM) refers to an array of interrelated competencies that enable human beings to understand one another's minds (Wellman, 2014). These competencies range from the early-developing ability to adopt others' visual perspectives (Sodian et al., 2007) to hard-won expertise inferring complex emotions from facial expressions (Eisenberg et al., 1997). Acting in concert, ToM competencies undergird our ability to trace others' behavior to their underlying beliefs, desires, and intentions (Apperly, 2012). ToM is thus a cornerstone of social cognition, a prerequisite for successful cooperation, and a universal developmental milestone (Astington et al., 1988; Malle et al., 2001). Not everyone, however, performs equally well on ToM tasks. For example, ToM is impaired in people with autism spectrum disorder (Yirmiya et al., 1998) and schizophrenia (Sprong et al., 2007). Performance on ToM tasks even varies among healthy adults - spurring researchers to investigate sources of systematic variation in people's ToM abilities (Apperly, 2012; Dumontheil et al., 2010; Keysar et al., 2003; Wellman, 2014).

A growing body of scholarship traces variation in ToM to differences in people's chronic levels of social attunement (Wu \& Keysar, 2007). One source of such differences is social class. Members of lower social classes, for example, are acculturated to prioritize the needs and preferences of others - and display superior performance on empathic accuracy tasks (Kraus et al., 2010, 2012). In the present paper, we present four studies (two initial investigations and two preregistered replications) that solidify and extend our understanding of the relationship between social class and ToM. We first address criticisms (Deveney et al., 2018) of previous work (Kraus et al., 2010) by robustly replicating the association between social class and emotion perception

(Studies 1A and 1B). We then extend these findings to a different facet of ToM, documenting for 
the first time a negative relationship between social class and visual perspective-taking (Studies 2A and 2B). We conclude by discussing related, yet distinct, pathways through which social class might affect different ToM competencies.

\section{Theory of Mind Competencies}

Theory of Mind encompasses a range of competencies that together enable people to solve problems in a world full of other minds. Examples of ToM include our ability to infer people's emotions, understand others' visual perspectives, trace behavior to actors' underlying intentions and desires, and grasp the fact that others' beliefs might misportray reality (Apperly, 2012; Apperly et al., 2008; Baron-Cohen et al., 1997; Flavell et al., 1981). We focus on two facets of ToM in this work: emotion perception and visual perspective-taking.

To successfully navigate the social world, individuals must be able to infer from people's faces what they are feeling - that is, engage in emotion perception. While the ability to recognize basic emotions (e.g., happiness and anger) may be innate (Arterberry et al., 2013), recognizing complex emotions (e.g., embarrassment and wistfulness) requires perceptual expertise developed over time. For complex emotions, individuals must acquire mental templates representing a large array of affective states and match them to incoming visual information (Baron-Cohen et al., 2001). The failure to correctly infer a complex emotion from a person's face may reflect either a lack of perceptual expertise (in the case of healthy adults) or an inability to understand the underlying emotional state (as in the case of individuals with autism).

Adults also possess ToM competencies less reliant on perceptual expertise. For example, most people are adept at taking others' visual perspectives — that is, at imagining what the world looks like from a location not their own. This critical ability allows perceivers to infer what 
others can and cannot see, and thus do and do not know. Visual perspective-taking is fully developed by the end of the preschool years (Moll \& Tomasello, 2006; Wellman et al., 2001) and can even be observed in some non-human primates (Call \& Tomasello, 2008). Yet despite the early and universal development of visual perspective-taking, adults may neglect to exercise this ability. Perspective-taking errors imply a failure to "select" another's perspective when one should — for instance, when correct judgment requires perceivers to appreciate the difference between what they can see and what others can see. Such failures can lead perceivers to misattribute their own knowledge to those not in a position to share it.

\section{The Cultural Roots of Variation in ToM}

Adults vary substantially in their performance on both emotion-reading and visual perspective-taking tasks (Baron-Cohen et al., 2001; Dumontheil et al., 2010). Nonetheless, the proximal causes of failures in these ToM domains appear to differ. In normal adults, errors identifying complex emotions likely reflect a lack of perceptual expertise, whereas errors in visual perspective-taking reflect a failure to properly execute a fully-developed capacity.

Despite their different immediate causes, failures across ToM domains may nonetheless stem from common distal factors. Perhaps the most important distal influence on a range of ToM competencies is culture (Luk et al., 2012; Markus \& Kitayama, 1991; Wu \& Keysar, 2007). The timing of ToM-related developmental milestones has been shown to vary between independent cultures, which prioritize community and attention to others, and interdependent cultures, which stress autonomy and attention to the self. For example, children in more interdependent cultures (e.g., Iran and China) understand that people who see something know about it ("knowledge 
access") at an earlier age than do children in more independent cultures (e.g., United States and Australia; Shahaeian et al., 2011; Slaughter \& Perez-Zapata, 2014; Wellman et al., 2006).

Culture is also associated with ToM performance in adulthood. To a greater extent than men, for example, women are acculturated to prioritize cooperation and community (Balliet et al., 2011; Eagly, 2009) — and consistently outperform men on tasks that require reading emotions from faces (Kirkland et al., 2013; Montagne et al., 2005). In the case of visual perspective-taking, individuals from East Asian countries, whose cultures emphasize social interdependence, tend to outperform Westerners on visual perspective-taking tasks (Wu \& Keysar, 2007). Such findings suggest that a habitual focus on others (as opposed to the self) is associated both with higher levels of ToM expertise and more efficient "online" deployment of ToM competencies in adults.

\section{Social Class and ToM Performance}

Although the majority of scholarship examining culture and ToM performance has focused on differences between national cultures (e.g., China vs. the United States; Wu \& Keysar, 2007), researchers have begun to apply the same logic to social class. Indeed, social classes are thought to represent distinct cultures that shape social life in fundamental ways, from health behaviors to styles of dress and food consumption (Darmon \& Drewnowski, 2008; Lachman \& Weaver, 1998). Lower social-class cultures are thought to foster interdependent values as an adaptation to environments poor in material resources, where successful functioning depends on social relationships and cooperation (Kraus et al., 2012; Piff, Stancato, Martinez, et al., 2012; Stephens et al., 2012). Higher social-class cultures, in contrast, foster independent strategies tailored to resource-rich environments, where successful functioning can be achieved 
with less help and cooperation from others (Kraus et al., 2012; Piff, Stancato, Martinez, et al., 2012; Stephens et al., 2012).

Reflecting their distinct value systems, members of different social-class cultures (e.g., working-class vs. upper middle-class individuals) vary in their attunement to other people (Dietze \& Knowles, 2016; Grossmann \& Varnum, 2011; Kraus \& Keltner, 2009; Stephens et al., 2014). For instance, compared to their higher-class counterparts, lower-class individuals tend to behave more prosocially (Piff, Stancato, Côté, et al., 2012), display more engagement cues during social interactions (Kraus \& Keltner, 2009), experience increased physiological arousal in response to others' suffering (Stellar et al., 2012), and devote more spontaneous visual attention to other humans (Dietze \& Knowles, 2016).

Dietze and Knowles (2016) argue that this array of findings concerning social class can be parsimoniously explained in terms of people's culturally-learned appraisals of others' motivational relevance - that is, rapid and spontaneous judgments of other people's significance. Compared to their higher-class counterparts, members of lower social classes are more likely to appraise other human beings as relevant to their current goals and well-being. As overlearned cultural defaults similar to what Bourdieu (1986) termed "habitus," class-based relevance appraisals are theorized to shape chronic and pervasive patterns of social cognition.

Minds are precisely what distinguish social from nonsocial entities. Thus, ToM-related aptitudes might be expected to vary between social classes that place differential emphasis on interdependence - and thus tend to appraise others as more or less motivationally relevant. Indeed, Kraus and colleagues (2010) found that performance on an array of empathic accuracy tasks varied inversely with perceivers' social class. In this research, for example, 
non-college-educated participants outperformed their college-educated counterparts on a standard test of emotion perception requiring that emotions be read from partial photographs of faces. As discussed in more detail below, however, these findings concerning social class and emotion reading - and, by implication, the claim that social class is inversely related to ToM more broadly — have been called into question (Deveney et al., 2018) and therefore require further examination.

\section{The Present Research}

The present studies aim to solidify and extend our knowledge of how Theory of Mind performance varies with social class. Using standard tests of two core ToM competencies - emotion perception and visual perspective-taking — we test the prediction that lower-class perceivers are better than their higher-class counterparts at reading complex emotions from facial expressions (Studies 1A and 1B) and at assuming the visual perspectives of other people (Studies 2A and 2B). In so doing, we attempt to reproduce a controversial finding regarding social class and emotion perception (Deveney et al., 2018; Kraus et al., 2010) and demonstrate for the first time that visual perspective-taking performance varies with social class. For each of the two ToM competencies, we report one initial study followed by an

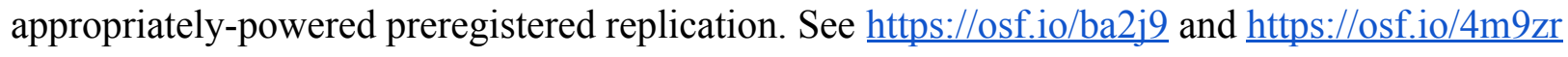
for preregistrations, and https://osf.io/z9xta and https://osf.io/jxbsm for full datasets and analysis code for all studies. 


\section{Study 1: Social Class and Emotion Perception}

Kraus and colleagues (2010) found that social class was inversely related to performance on a standard test of emotion perception — specifically, the Reading the Mind in the Eyes Test (RMET; Baron-Cohen et al., 2001). Yet recent research has called this finding into question. In three large online samples, Deveney and colleagues (2018) failed to replicate the negative relationship between social class and emotion-perception accuracy in the RMET and related tasks. The researchers conclude that Kraus et al.'s (2010) results may not generalize beyond a narrow range of participant samples (i.e., students and employees at a large public university).

Given the tension between Kraus et al.’s (2010) and Deveney et al.'s (2018) findings, the nature of the association between social class and emotion perception ability is an open question requiring independent adjudicating evidence. Thus, in Study 1, we test (Study 1A) and replicate (Study 1B) the negative relationship between social class and emotion-perception performance using the RMET.

\section{Study 1A}

\section{Participants}

Participants were 300 workers (137 male, 160 female, 3 other) on Amazon's Mechanical Turk crowdsourcing platform (Buhrmester et al., 2011), aged 18 to $69(\mathrm{M}=35.45, \mathrm{SD}=11.21)$. All participants were United States nationals. 225 participants identified as White, 29 as African American, 19 as Latino/a, 10 as East Asian/Asian American, and 6 as Native American, with 11 participants specifying another ethnicity. No data were excluded from analysis. 


\section{Materials and Procedure}

Emotion perception performance was assessed using the Reading the Mind in the Eyes Test (RMET; Baron-Cohen et al., 2001; Kraus et al., 2010). In the RMET, participants view 36 pictures displaying the eyes and surrounding areas of a series of faces. Each picture portrays a different emotion (e.g., worried, interested, hostile). For each stimulus, participants are shown a list of 4 emotion adjectives and must choose the adjective that best matches the pictured emotion. To account for individual differences in facility with emotion vocabulary, participants can opt to display the definition of each emotion word. The number of correct answers is summed for each participant, with higher scores indicating more accurate emotion perception.

As in previous research (Dietze \& Knowles, 2016), and consistent with the notion that ToM abilities are influenced by cultural factors related to social class, we selected a group-based measure of class (see Dietze \& Knowles, 2016, for a discussion of alternative measures of social class and their differential relevance to the cultural dimensions of class). We used the same social class measure across all studies (and analyses) presented in this manuscript. The social class category probe read as follows: "People talk about social classes such as the poor, the working class, the middle class, the upper-middle class, and the upper class. Which of these classes would you say you belong to?" This measure has been shown to intuitively and meaningfully capture group-based class distinctions in the United States (Dietze \& Knowles, 2016; Jackman \& Jackman, 1983). Participants' responses were converted to an ordinal variable ranging from 1 (poor) to 5 (upper class). A number of additional measures were included for exploratory purposes and administered after the social class category probe (see supplement for more information). 


\section{Results}

We hypothesized that scores on the RMET would vary inversely with participants' social class category. To test this, we examined the bivariate Pearson's correlation between the ordinal class category variable and RMET scores. Consistent with our prediction, this negative correlation was significant $(r=-.169, p=.003,95 \% \mathrm{CI}[-.277,-.057])$. See Figure 1 (left panel) for a visual depiction of the class-RMET relationship in Study 1A.

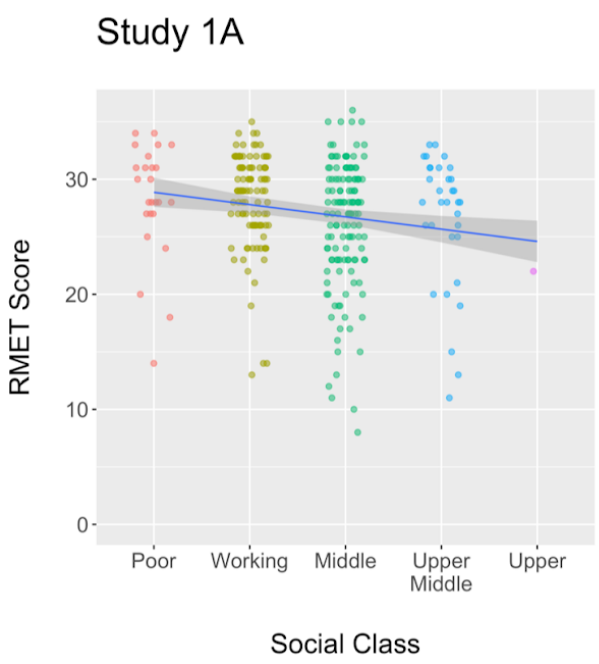

\section{Study 1B}

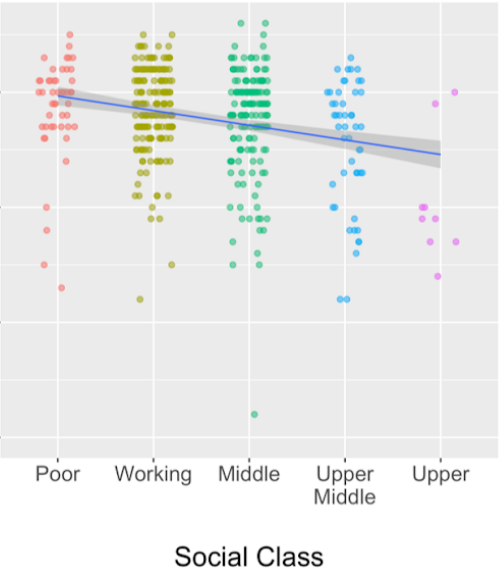

Integrated Dataset

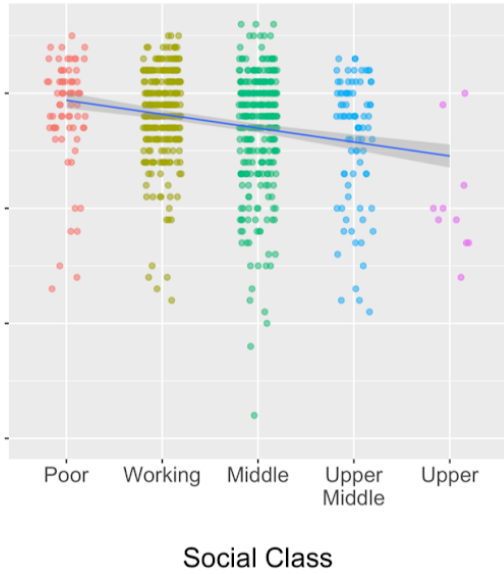

Figure 1. Reading the Mind in the Eyes Test (RMET) score as a function of social class category in Study 1A (left panel), Study 1B (middle panel), and the integrated dataset (right panel). Dots $=$ individual participants; lines $=$ linear regression results; error bands $=95 \%$ confidence envelopes.

\section{Study 1B}

Study 1B was a preregistered replication of Study 1A using identical procedures and materials. In Study 1B, however, participants were drawn from the Prolific Academic crowdsourcing platform (Peer et al., 2017) instead of Amazon Mechanical Turk, allowing us to 
generalize the results across online data-collection platforms. Preregistration materials are posted on the Open Science Framework (OSF; https://osf.io/ba2j9).

\section{Participants}

Power analysis revealed that a sample of 450 participants would yield a $95 \%$ chance of replicating the previously observed relationship between social class and RMET scores. We recruited 451 workers (239 male, 210 female, 2 other) from the Prolific Academic online research platform, aged 18 to $78(\mathrm{M}=33.77, \mathrm{SD}=11.47)$. All participants were U.S. nationals. 313 participants identified as White, 34 as African American, 31 as Latino/a, 32 as East Asian/Asian Americans, and 17 as Native American, with 24 participants specifying another ethnicity or declining to answer the question. No data were excluded from analysis.

\section{Materials and Procedure}

As in Study 1, the Reading the Mind in the Eyes Test (RMET) was used to assess emotion perception and the social class category probe (Dietze \& Knowles, 2016; Jackman \& Jackman, 1983) was used to assess social class membership. Additional measures were included for exploratory purposes and administered after the social class category probe (see supplement for more information).

\section{Results}

We again hypothesized that scores on the RMET would vary inversely with participants' social class category. Consistent with our prediction, RMET scores were negatively correlated with participants' social class category $\left(r=-.250, p=7.297 \times 10^{-8}, 95 \% \mathrm{CI}[-.335,-.162]\right)$. See Figure 1 (middle panel) for a visual depiction of the class-RMET relationship in Study 1 B. 


\section{Integrative Data Analysis (Studies 1A and 1B)}

Using Integrative Data Analysis (IDA), we sought to synthesize our research findings across the original and replication samples while also quantifying between-sample heterogeneity (Curran \& Hussong, 2009). Like meta-analysis, IDA is a useful technique for integrating results across studies; IDA, however, is the preferred approach when all original data are available. In IDA, hypotheses are tested using the combined datasets. Between-study heterogeneity is modeled either as a random effect or, when few individual datasets are available, as a fixed effect. Because we are integrating only two studies, a fixed-effects IDA was used (Curran \& Hussong, 2009). We therefore combined the Study 1A and Study 1B data, effect-coded study (-1 $=$ Study $1 \mathrm{~A}, 1=$ Study $1 \mathrm{~B})$, and reran our analyses_allowing study to predict RMET scores as a main effect and in interaction with the social class category probe. As can be seen in Table 1, the observed and replicated relationship between social class and emotion perception was robust and homogeneous across studies (and online research platforms): Lower-class participants tended to score higher on the RMET than did high-class participants $(\mathrm{B}=-1.170, \mathrm{SE} \mathrm{B}=0.208, t=-5.630$, $\left.p=2.55 \times 10^{-8}, 95 \% \mathrm{CI}[-1.578,-0.762]\right)$. See Figure 1 (right panel) for a visual depiction of the class-RMET relationship across studies.

Table 1. Integrative data analysis of RMET scores as a function of social class category and study.

\begin{tabular}{l|cccccc}
\hline \multicolumn{1}{c|}{ Predictor } & \multicolumn{1}{c}{} & & \multicolumn{2}{c}{$95 \% \mathrm{Cl}$} \\
& B & SE B & $t$ & $p$ & LB & UB \\
\hline Study & 0.201 & 0.195 & 1.031 & 0.303 & -0.182 & 0.584 \\
Social Class Category (SCC) & $\mathbf{- 1 . 1 7 0}$ & $\mathbf{0 . 2 0 8}$ & $\mathbf{- 5 . 6 3 0}$ & $\mathbf{2 . 5 5 \times \mathbf { 1 0 }}$ & $\mathbf{- 1 . 5 7 8}$ & $\mathbf{- 0 . 7 6 2}$ \\
Study $\times$ SSC & -0.103 & 0.208 & -0.495 & 0.621 & -0.511 & 0.305 \\
\hline
\end{tabular}

Note. Study coded such that $-1=$ Study 1 A and $1=$ Study 1 B. Social class category centered at "middle class." 


\section{Robustness Against Confounds}

Any confounding of social class with other participant demographics would undermine our argument for the existence of social-class differences in emotion perception. Indeed, we find that social class is correlated with participants' race; in Studies 1A and 1B, Blacks and "other race" participants tended to be lower in social class than their White counterparts (Supplemental Table S1). In light of this association between class and race, we repeated our analysis of RMET scores as a function of social class in the combined dataset - this time adding dummy-coded race vectors as covariates. Because past research documents associations between age, gender, and ToM performance (Dumontheil et al., 2010; Kirkland et al., 2013), gender and age covariates were also added to the model. Inclusion of these covariates did not substantially alter the relationship between social class and RMET scores $(\mathrm{B}=-1.296, \mathrm{SE} \mathrm{B}=0.197, t=-6.268, p=$ $1.002 \times 10^{-10}, 95 \%$ CI[-1.683, -0.908]; Supplemental Table S2).

\section{Discussion}

Study 1 provides robust and replicable evidence for an inverse relationship between social class and the core Theory of Mind competency of emotion perception. Across two online samples, people outperformed their higher-class counterparts on a well-established test of “advanced" emotion perception (Baron-Cohen et al., 2001) — the Reading the Mind in the Eyes Test (Baron-Cohen et al., 2001). Because the RMET taps individual differences in perceptual expertise, it appears that lower-class perceivers' visual representations of complex emotions may be better-specified than those of their higher-class counterparts.

In the second pair of studies, we extend our reasoning concerning social class and Theory of Mind from emotion perception to another critical ToM competency: visual perspective-taking. 
Although both competencies are "inferential" (Apperly et al., 2008), in that participants must infer (i.e., are not told) what another person feels or sees, the tasks differ in an important respect. On the one hand, the inference of complex emotions requires ability; individuals are limited in their performance by the fixed ceiling of their perceptual expertise. Visual perspective-taking, in contrast, requires execution; all normal adults can enact this fully-developed ability, but may nonetheless fail to do so when correct judgment requires it (Wu \& Keysar, 2007).

Perspective-taking thus affords an opportunity to investigate whether social class predicts the online deployment of a universal ToM competency.

\section{Study 2: Social Class and Visual Perspective-Taking}

The purpose of Study 2 is to extend our understanding of the social class-ToM relationship to another facet of Theory of Mind: visual perspective-taking. A primary function of perspective-taking is to disambiguate utterances made by speakers whose vantage differs from our own. To illustrate, imagine two friends seated across from one another at a dinner table. If one friend asks the other to "pass the spoon," and there are two spoons on the table, then this utterance is ambiguous. Suppose, however, that a large serving bowl blocks the requester's view of one of the spoons. By imaginatively adopting the visual perspective of her friend, the requestee may infer that the requester cannot see the occluded spoon — and therefore must want the other one. This logic forms the basis of a standard test of visual perspective-taking performance in adults, the Director Task, in which correct judgments require adopting another's point of view (Keysar, 1997; Keysar et al., 2003).

While all normal adults can perspective-take, they sometimes fail to do so when they should. Performance on the Director Task, in particular, appears to vary as a function of 
participants' national culture (Luk et al., 2012; Wu \& Keysar, 2007), raising the possibility that social class might also predict differences on the task. Consistent with the idea that higher levels of social attunement are associated with superior ToM performance, we hypothesized that members of lower social classes would be less likely to display failures of perspective-taking in the Director Task than would their higher-class counterparts. Study 2A provides an initial test of this hypothesis and Study 2B replicates Study 2A's results.

\section{Study $2 A$}

\section{Participants}

Sixty-six undergraduates at New York University were recruited for Study 2A. Only Native English-speakers were eligible to participate. Six participants had to be excluded from analysis because they had no data-five due to a technical malfunction during the task and one due to missing questionnaire data. The final sample consisted of 59 participants (29 male, 30 female), aged 18 to $23(\mathrm{M}=19.7, \mathrm{SD}=1.42)$. Twenty-two participants identified as White, 4 as African American, 10 as Latino/a, 15 as East Asian/Asian Americans, with eight participants specifying another ethnicity or declining to answer the question.

\section{Materials and Procedure}

In order to assess visual perspective-taking performance, we used a computerized version of the Director Task. The procedure and stimuli were adapted from a previous study (Dumontheil et al., 2010). In the task, participants see an array of compartments arranged in a 4 $\times 4$ grid (Figure 2). A subset of the compartments contain objects, some of which must be moved according to spoken instructions. Each participant is exposed to a director and no-director condition in sequential order. 


\section{Director Condition}

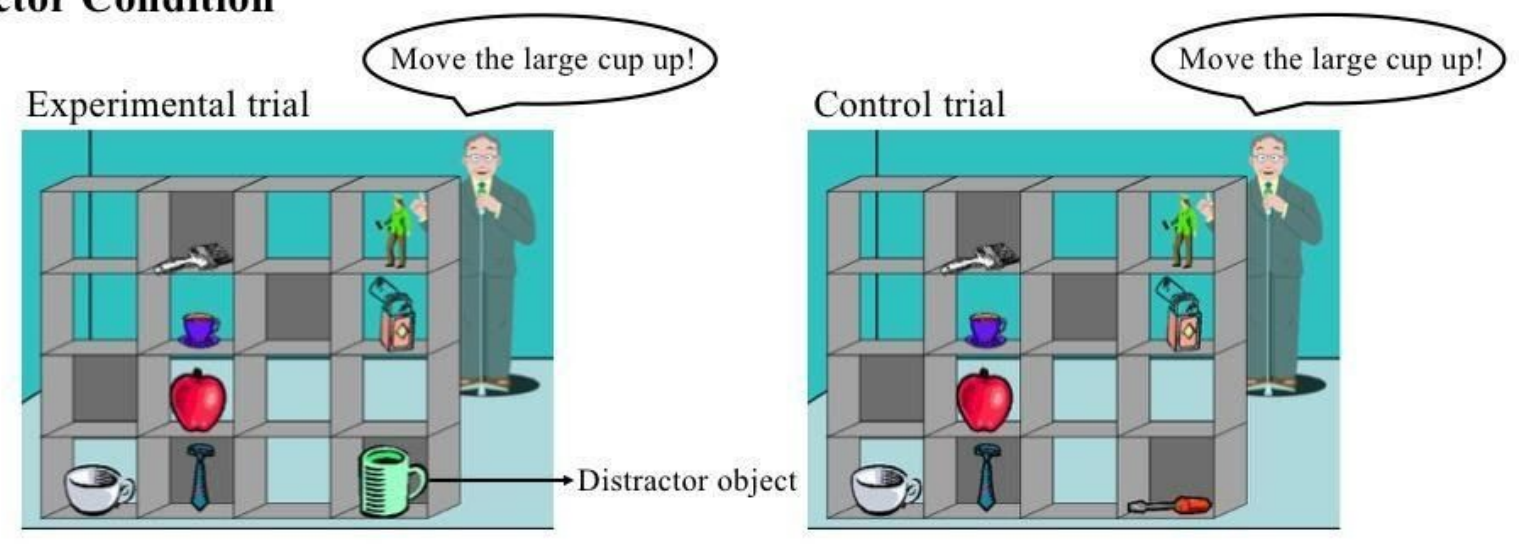

\section{No-Director Condition}

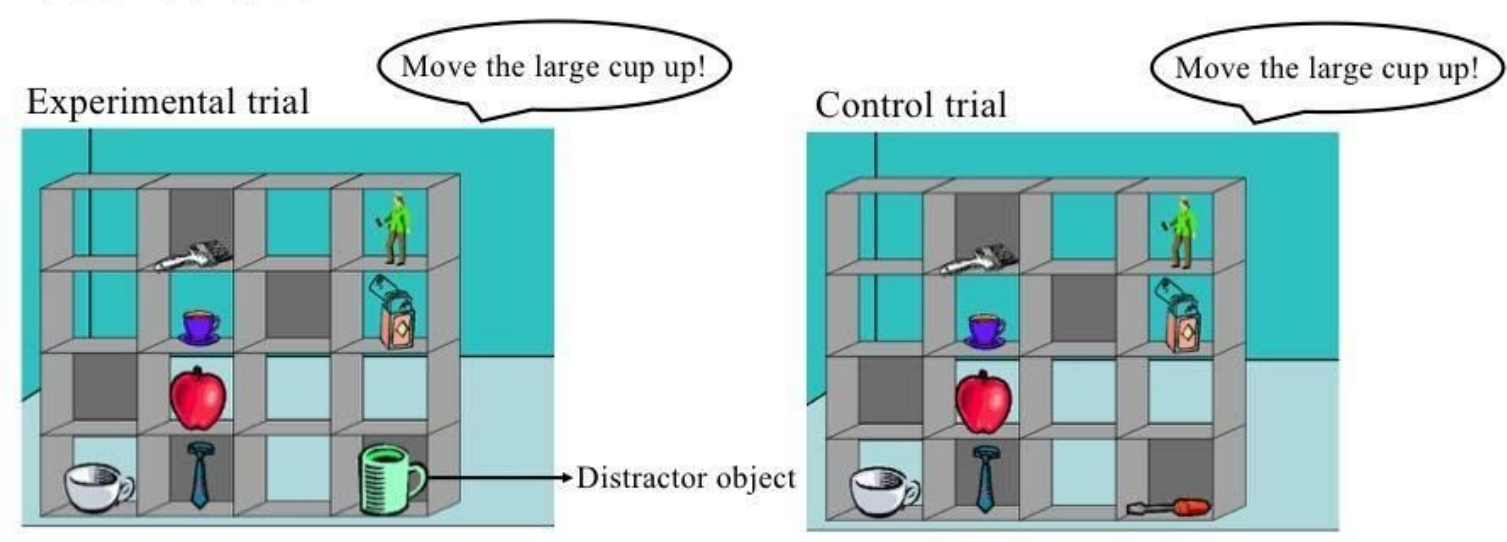

Figure 2. Example of experimental and control trials in the director and no-director conditions. Experimental trials display a distractor object (green cup) whereas control trials display an irrelevant object (screwdriver). In the director condition, experimental trials measure ToM performance. Failure to take the director's perspective would mean choosing the distractor object (green cup) instead of the correct object (white cup).

In the director condition, a human avatar stands on the far side of the grid — assuming a perspective opposite to that of the participant—and gives instructions to move objects. Some objects, however, are occluded from the director's view. In order to successfully follow the director's instructions, participants must take into account the director's compromised view of the grid compartments. Participants complete 12 experimental trails, 12 control trials, and 24 filler trials in random order. 
On experimental trials, the director gives instructions that could refer to an object visible to the participant but occluded from the director's view (i.e., a competitor object; Figure 2). If the participant disregards the director's view and takes an egocentric perspective, then the participant is likely to pick the wrong object (e.g., the green cup only visible to the participant; Figure 2). Control trials are identical to experimental trials except that the competitor object (e.g., the green cup) is replaced by an irrelevant object (e.g., a screwdriver). Thus, in control trials, the item to be moved is unambiguous. On filler trials, the instructions refer to a single object visible to both the director and the participant (e.g., "move the apple up").

The no-director condition controls for the general cognitive demands of the task. In this condition, the director is absent and the participant is given a simple rule to follow: ignore all objects that appear against a dark grey background. Participants again complete 12 experimental trials, 12 control trials, and 24 filler trials in random order. On experimental trials, the participant receives instructions that could refer to a competitor object that appears against a dark grey background. Control trials are identical to experimental trials, except that the competitor object has been replaced by an irrelevant object. On filler trials, the instructions refer to a single object that appears against a white background.

Participants are instructed to click on the object to which the instructions refer on each trial. All instructions were delivered via headphones. In the experimental condition, errors were defined as the participant selecting the competitor object rather than the target; ${ }^{1}$ in the control condition, errors were defined as selecting any object other than the target. Response times were measured in all conditions.

\footnotetext{
${ }^{1}$ Alternatively, errors in the experimental condition could be defined as selecting any object other than the target. Our results remain substantively unchanged when this coding scheme is used.
} 
After finishing the Director Task, participants completed the social class category probe used in Studies 1A and 1B (Dietze \& Knowles, 2016). As before, our analyses centered on this measure of social class, but additional measures were included for exploratory purposes and administered after the social class category probe (see supplement for more information).

\section{Results}

In the Director Task, failures of perspective taking can be inferred when participants make disproportionately more errors identifying objects in the presence of both the human director and a competitor object. Thus, perspective-taking failures imply a two-way interactive effect of the presence (vs. absence) of the director and the presence (vs. absence) of a competitor object on identification errors.

Our first analysis tested whether the sample as a whole exhibited perspective-taking failures. Because the data are nested, with 96 trials per participant, we conducted a mixed-effects logistic regression to examine the effects of director $(-1=$ no director, $1=$ director present), competitor $(-1=$ no competitor, $1=$ competitor present $)$, and the Director $\times$ Competitor interaction, on the per-trial probability of an identification error. Random intercepts and slopes were specified for both independent variables, and covariances between these random effects were freely estimated. Results revealed significant main effects of director $(\mathrm{B}=0.824, \mathrm{SE} B=$ $0.216, z=3.814, p<.001,95 \% \mathrm{CI}[0.401,1.248])$ and competitor $(\mathrm{B}=1.174, \mathrm{SE} \mathrm{B}=0.216, z=$ $5.440, p<.001,95 \%$ CI $[0.751,1.596])$, and — critically — a significant Director $\times$ Competitor interaction $(\mathrm{B}=0.671, \mathrm{SE} \mathrm{B}=0.140, z=4.778, p<.001,95 \% \mathrm{CI}[0.396,0.946])$. Examining predicted marginal probabilities, we find that participants rarely made identification errors in the absence of either a director or a competitor (no director and no competitor: estimate $=.026, S E=$ 
$.008,95 \% \mathrm{CI}[0.010,0.042]$; director but no competitor: estimate $=.042, S E=.013,95 \% \mathrm{CI}$

$[0.017,0.067]$; competitor but no director: estimate $=.130, S E=.030,95 \% \mathrm{CI}[0.071,0.189])$. In contrast, the presence of both the director and a competitor object led to a sharp increase in identification errors (estimate $=.429, S E=.046,95 \% \mathrm{CI}[0.338,0.520]$ ), indicating that participants were vulnerable to perspective-taking failures in the Director Task.

We hypothesized that participants higher in social class would make more perspective-taking errors than would their lower-class counterparts. We therefore expected the Director $\times$ Competitor interaction described above to be more pronounced among higher-class participants than among lower-class participants. To test this, we conducted a mixed-effects logistic regression examining the effects of social class (centered at "middle class"), director (-1 $=$ no director, $1=$ director present $)$, competitor $(-1=$ no competitor, $1=$ competitor present $)$, and all two-and three-way interactions between these variables, on the per-trial probability of an identification error. Random intercepts and slopes were specified for director and competitor, and covariances between these random effects were freely estimated. Complete regression results are shown in Table 2. 
Table 2. Mixed-effects logistic regression analysis of identification errors in the Director Task (Study 2A).

\begin{tabular}{|c|c|c|c|c|c|c|}
\hline \multirow[b]{2}{*}{ Predictor } & \multirow[b]{2}{*}{ B } & \multirow[b]{2}{*}{ SE B } & \multirow[b]{2}{*}{$z$} & \multirow[b]{2}{*}{$p$} & \multicolumn{2}{|c|}{$95 \% \mathrm{Cl}$} \\
\hline & & & & & LB & UB \\
\hline Social Class Category (SSC) & 0.235 & 0.221 & 1.064 & 0.287 & -0.198 & 0.668 \\
\hline Director (D) & 0.653 & 0.225 & 2.899 & 0.004 & 0.211 & 1.094 \\
\hline Competitor (C) & 0.922 & 0.222 & 4.143 & $3.435 \times 10^{-5}$ & 0.486 & 1.358 \\
\hline$S S C \times D$ & 0.212 & 0.176 & 1.204 & 0.229 & -0.133 & 0.558 \\
\hline $\mathrm{SSC} \times \mathrm{C}$ & 0.425 & 0.186 & 2.284 & 0.022 & 0.060 & 0.790 \\
\hline$D \times C$ & 0.603 & 0.144 & 4.198 & $2.689 \times 10^{-5}$ & 0.321 & 0.885 \\
\hline$S S c \times D \times C$ & 0.237 & 0.103 & 2.313 & 0.021 & 0.036 & 0.438 \\
\hline
\end{tabular}

Note. Director and competitor coded such that $-1=$ no director or competitor object and $1=$ director or competitor object present. Social class centered at "middle class." The SSC $\times \mathrm{D} \times \mathrm{C}$ interaction reflects more perspective-taking failures among higher-class participants than among lower-class participants.

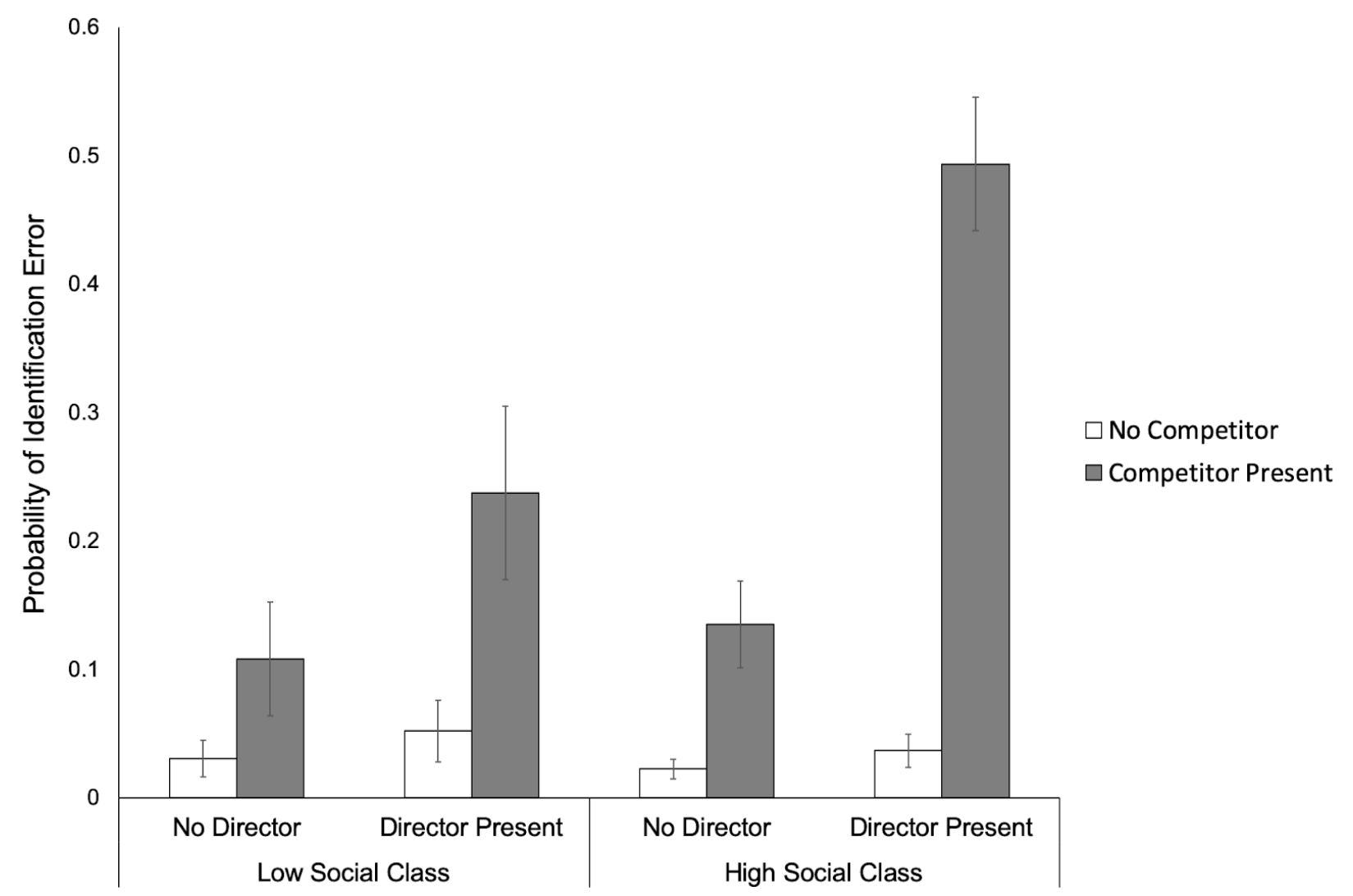

Figure 3. Object-identification error probability as a function of participant social class, the presence of a human director, and the presence of a competitor object (Study 2A). Error bars represent $95 \%$ confidence intervals. 
As predicted, we observed a significant Social Class Category $\times$ Director $\times$ Competitor interaction. Figure 3 displays predicted error probabilities as a function of participant social class, presence of the director, and presence of a competitor object. The significant three-way interaction reflects the fact that the Director $\times$ Competitor interaction was more pronounced among higher-class participants than among lower-class participants. A focused test of the Director $\times$ Competitor interaction among lower-class participants (defined for graphing purposes as "working class") did not reach significance $(\mathrm{B}=0.366, \mathrm{SE} \mathrm{B}=0.195, \mathrm{z}=1.878, \mathrm{p}=.060$, 95\% CI [-0.198, 0.668]); however, the Director $\times$ Competitor interaction among higher-class participants (defined for graphing purposes as "upper-middle class") was highly significant (B = $0.840, \mathrm{SE} \mathrm{B}=0.156, z=5.384, \mathrm{p}<.001,95 \% \mathrm{CI}[0.534,1.146])$. These results indicate higher rates of perspective-taking failure among those higher (vs. lower) in social class.

\section{Study 2B}

In Study 2B, we aimed to reproduce the findings of Study $2 \mathrm{~A}$ in a preregistered replication. The study procedure and measures were identical. Preregistration materials are posted on the Open Science Framework (OSF; https://osf.io/4m9zr).

\section{Participants}

Seventy-two undergraduates at New York University were recruited for Study 2B-enough to yield 95\% power based on the effect sizes in Study 2A. Only Native English-speakers were eligible to participate. Two participants had no data (one due to a technical malfunction and one a lack of questionnaire data) and were excluded from analysis. The final sample thus consisted of 70 participants (22 male, 48 female), aged 17 to $22(\mathrm{M}=$ 18.91, $\mathrm{SD}=1.05)$. Twenty-five participants identified as White, 8 as African American, 9 as 
Latino/a, 24 as East Asian/Asian Americans, and 4 participants specifying another ethnicity or declining to answer the question.

\section{Materials and Procedures}

The materials and procedure of Study $2 \mathrm{~B}$ were identical to those of Study 2A, with the Director Task used to assess visual perspective-taking and social class category probe (Dietze \& Knowles, 2016) to assess social class. Additional measures were included for exploratory purposes and administered after the social class category probe (see supplement for more information).

\section{Results}

The purpose of Study 2B was to replicate the Social Class Category $\times$ Director $\times$ Competitor interaction observed in Study 2A. The replication data were structured identically to those of the original study; thus, the same analyses were conducted.

We first tested whether our replication sample exhibited perspective-taking failures. Results of a mixed-effects logistic regression revealed main effects of director $(B=1.263, \mathrm{SE} B$ $=0.243, z=5.205, p<.001,95 \% \mathrm{CI}[0.788,1.739])$ and competitor $(\mathrm{B}=1.611, \mathrm{SE} \mathrm{B}=0.317, z$ $=5.086, p<.001,95 \% \mathrm{CI}[0.990,2.232])$, as well as a significant Director $\times$ Competitor interaction $(\mathrm{B}=0.390, \mathrm{SE} \mathrm{B}=0.116, z=3.359, p=.001,95 \% \mathrm{CI}[0.162,0.617])$. Examining predicted marginal probabilities, we found that participants made relatively few identification errors in the absence of either a director or competitor (no director and no competitor: estimate $=$ $.136, S E=.034,95 \%$ CI $[0.069,0.203]$; director but no competitor: estimate $=.119, S E=.029$, $95 \%$ CI $[0.063,0.176]$; competitor but no director: estimate $=.200, S E=.040,95 \%$ CI $[0.122$, 0.279]). In contrast, the presence of both the director and a competitor object led to an increase 
identification errors (estimate $=.436, S E=.043,95 \% \mathrm{CI}[0.351,0.521]$ ), indicating that, as in the previous study, participants displayed perspective-taking failures in the Director Task.

We again expected the interaction between director and competitor to be more pronounced among higher-class participants than among lower-class participants. Results of a mixed effects regression revealed the predicted Social Class $\times$ Director $\times$ Competitor interaction (Table 3). Figure 4 displays predicted error probabilities as a function of participant social class, presence of the director, and presence of a competitor object. As in Study 2A, the Social Class $\times$ Director $\times$ Competitor reflects the fact that the Director $\times$ Competitor interaction was more pronounced among higher-class participants than among lower-class participants. A focused test of the Director $\times$ Competitor interaction among lower-class participants (defined for graphing purposes as "working class") did not reach significance $(\mathrm{B}=-0.151, \mathrm{SE} \mathrm{B}=.226, z=0.570, p=$ $.569,95 \% \mathrm{CI}[-0.099,1.914])$; however, the Director $\times$ Competitor interaction among higher-class participants (defined for graphing purposes as "upper-middle class") was highly significant $(\mathrm{B}=0.520, \mathrm{SE} \mathrm{B}=.129, z=4.038, p<.001,95 \% \mathrm{CI}[0.267,0.772])$. These results indicate higher rates of perspective-taking failure among those higher (vs. lower) in social class - thus replicating the key result of Study 2A. 
Table 3. Mixed-effects logistic regression analysis of identification errors in the Director Task (Study 2B).

\begin{tabular}{|c|c|c|c|c|c|c|}
\hline \multirow[b]{2}{*}{ Predictor } & \multirow[b]{2}{*}{ B } & \multirow[b]{2}{*}{ SE B } & \multirow[b]{2}{*}{$z$} & \multirow[b]{2}{*}{$p$} & \multicolumn{2}{|c|}{$95 \% \mathrm{Cl}$} \\
\hline & & & & & LB & UB \\
\hline Social Class Category (SSC) & 0.908 & 0.513 & 1.768 & 0.077 & -0.099 & 1.914 \\
\hline Director (D) & 1.455 & 0.284 & 5.127 & $2.945 \times 10^{-7}$ & 0.899 & 2.012 \\
\hline Competitor (C) & 1.872 & 0.368 & 5.090 & $3.578 \times 10^{-7}$ & 1.151 & 2.592 \\
\hline$S S C \times D$ & -0.270 & 0.244 & -1.110 & 0.267 & -0.748 & 0.207 \\
\hline$S S C \times C$ & -0.562 & 0.344 & -1.632 & 0.103 & -1.236 & 0.113 \\
\hline$D \times C$ & 0.184 & 0.147 & 1.252 & 0.211 & -0.104 & 0.473 \\
\hline$S S C \times D \times C$ & 0.336 & 0.148 & 2.268 & 0.023 & 0.046 & 0.625 \\
\hline
\end{tabular}

Note. Director and competitor coded such that $-1=$ no director or competitor object and $1=$ director or competitor object present. Social class centered at "middle class." The SSC $\times \mathrm{D} \times \mathrm{C}$ interaction reflects more perspective-taking failures among higher-class participants than among lower-class participants.

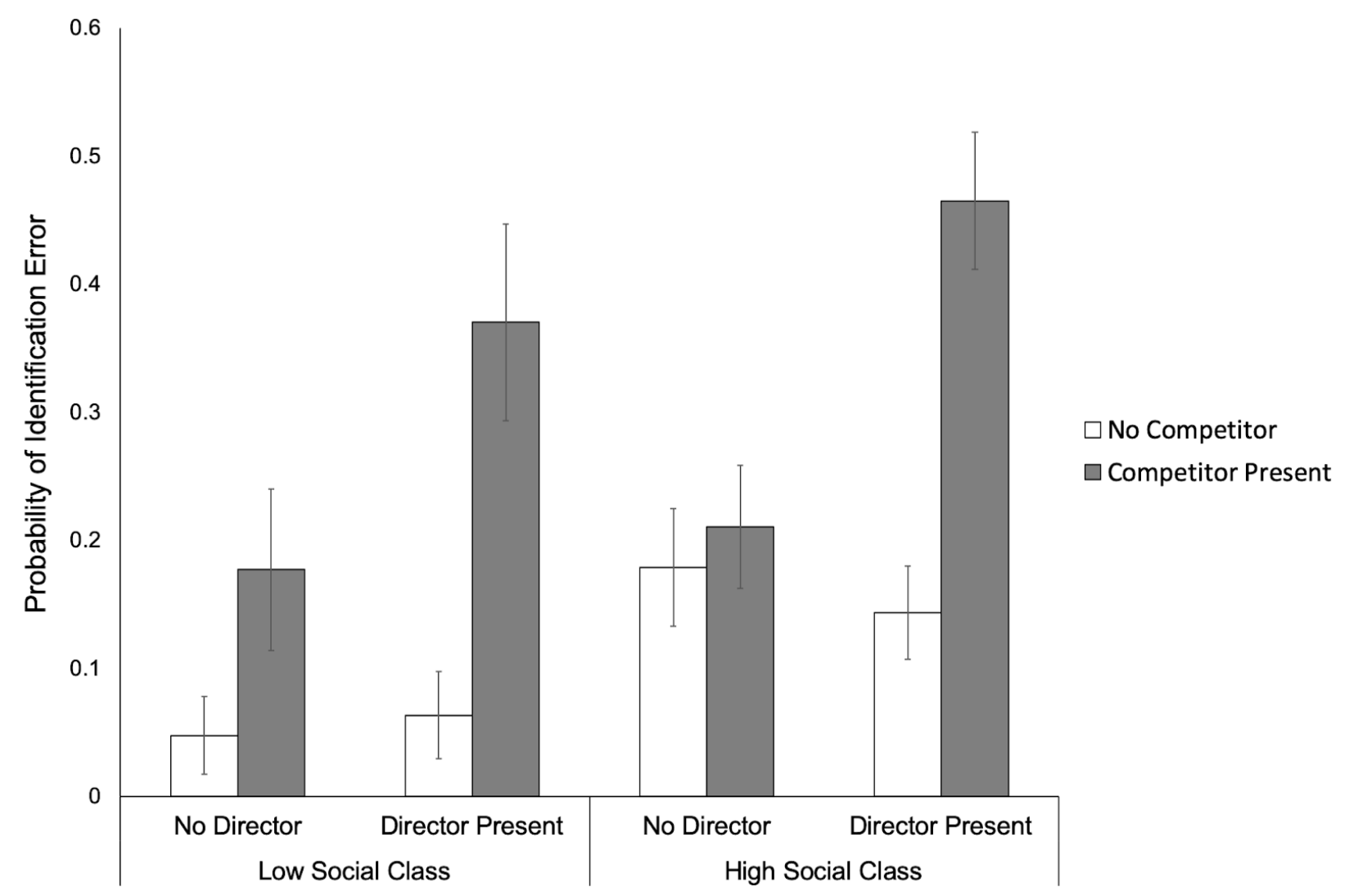

Figure 4. Object-identification error probability as a function of participant social class, the presence of a human director, and the presence of a competitor object (Study 2B). Error bars represent $95 \%$ confidence intervals. 


\section{Integrative Data Analysis (Studies 2A and 2B)}

Using Integrative Data Analysis (IDA), we sought to synthesize our research findings across the original and replication samples and quantify between-sample heterogeneity (Curran \& Hussong, 2009). We therefore combined the Studies 2A and 2B data, effect-coded study $(-1=$ Study 2A, 1 = Study 2B), and reran our analyses - allowing study to predict object identification errors as a main effect and in interaction with all other predictor variables (i.e., social class, director, and competitor). Full regression results are displayed in Table 4.

As can be seen in Table 4, only the Social Class $\times$ Competitor interaction differed significantly across the original and replication samples. However, this interaction is not probative of the association between social class and perspective-taking failures. The Social Class $\times$ Director $\times$ Competitor interaction, in contrast, does reflect this association — and did not differ across samples. Thus, across Studies 2A and 2B, members of higher social classes displayed a more pronounced Director $\times$ Competitor interaction $(\mathrm{B}=0.652, \mathrm{SE} \mathrm{B}=0.100, z=$ $6.495, p<.001,95 \%$ CI $[.456, .849])$ than did their lower-class counterparts $(\mathrm{B}=0.130, \mathrm{SE} \mathrm{B}=$ $0.163, z=0.798, p=.425,95 \%$ CI $[-.422, .598]$; see Figure 5 for predicted error probabilities). 
Table 4. Mixed-effects logistic regression analysis of identification errors in the Director Task (combined Study 2A and Study 2B data).

\begin{tabular}{|c|c|c|c|c|c|c|}
\hline \multirow[b]{2}{*}{ Predictor } & \multirow[b]{2}{*}{ B } & \multirow[b]{2}{*}{ SE B } & \multirow[b]{2}{*}{$z$} & \multirow[b]{2}{*}{$p$} & \multicolumn{2}{|c|}{$95 \% \mathrm{Cl}$} \\
\hline & & & & & LB & UB \\
\hline Study (S) & 0.088 & 0.260 & 0.337 & 0.736 & -0.422 & 0.598 \\
\hline Social Class Category (SCC) & 0.500 & 0.250 & 2.002 & 0.045 & 0.010 & 0.990 \\
\hline Director (D) & 1.061 & 0.177 & 6.002 & $1.947 \times 10^{-9}$ & 0.715 & 1.408 \\
\hline Competitor (C) & 1.410 & 0.202 & 6.982 & $2.906 \times 10^{-12}$ & 1.014 & 1.806 \\
\hline$S \times S C C$ & 0.262 & 0.249 & 1.049 & 0.294 & -0.227 & 0.750 \\
\hline$S \times D$ & 0.123 & 0.152 & 0.813 & 0.416 & -0.174 & 0.421 \\
\hline$S \times C$ & 0.118 & 0.185 & 0.635 & 0.525 & -0.245 & 0.480 \\
\hline$S C C \times D$ & -0.019 & 0.146 & -0.130 & 0.897 & -0.305 & 0.267 \\
\hline $\mathrm{SCC} \times \mathrm{C}$ & 0.026 & 0.180 & 0.146 & 0.884 & -0.326 & 0.379 \\
\hline$D \times C$ & 0.391 & 0.102 & 3.856 & $1.155 \times 10^{-4}$ & 0.192 & 0.590 \\
\hline$S \times S C C \times D$ & -0.200 & 0.145 & -1.377 & 0.169 & -0.484 & 0.085 \\
\hline $\mathrm{S} \times \mathrm{SCC} \times \mathrm{C}$ & -0.443 & 0.181 & -2.453 & 0.014 & -0.797 & -0.089 \\
\hline$S \times D \times C$ & -0.083 & 0.088 & -0.936 & 0.349 & -0.256 & 0.090 \\
\hline$S C C \times D \times C$ & 0.261 & 0.090 & 2.902 & 0.004 & 0.085 & 0.437 \\
\hline$S \times S C C \times D \times C$ & 0.041 & 0.090 & 0.454 & 0.650 & -0.136 & 0.218 \\
\hline
\end{tabular}

Note. Study coded such that $-1=$ Study $2 \mathrm{~A}$ and $1=$ Study $2 \mathrm{~B}$. Director and competitor coded such that $-1=$ no director or competitor object and $1=$ director or competitor object present. Social class category centered at "middle class." The SSC $\times$ D $\times$ C interaction reflects more perspective-taking failures among higher-class participants than among lower-class participants across studies. 


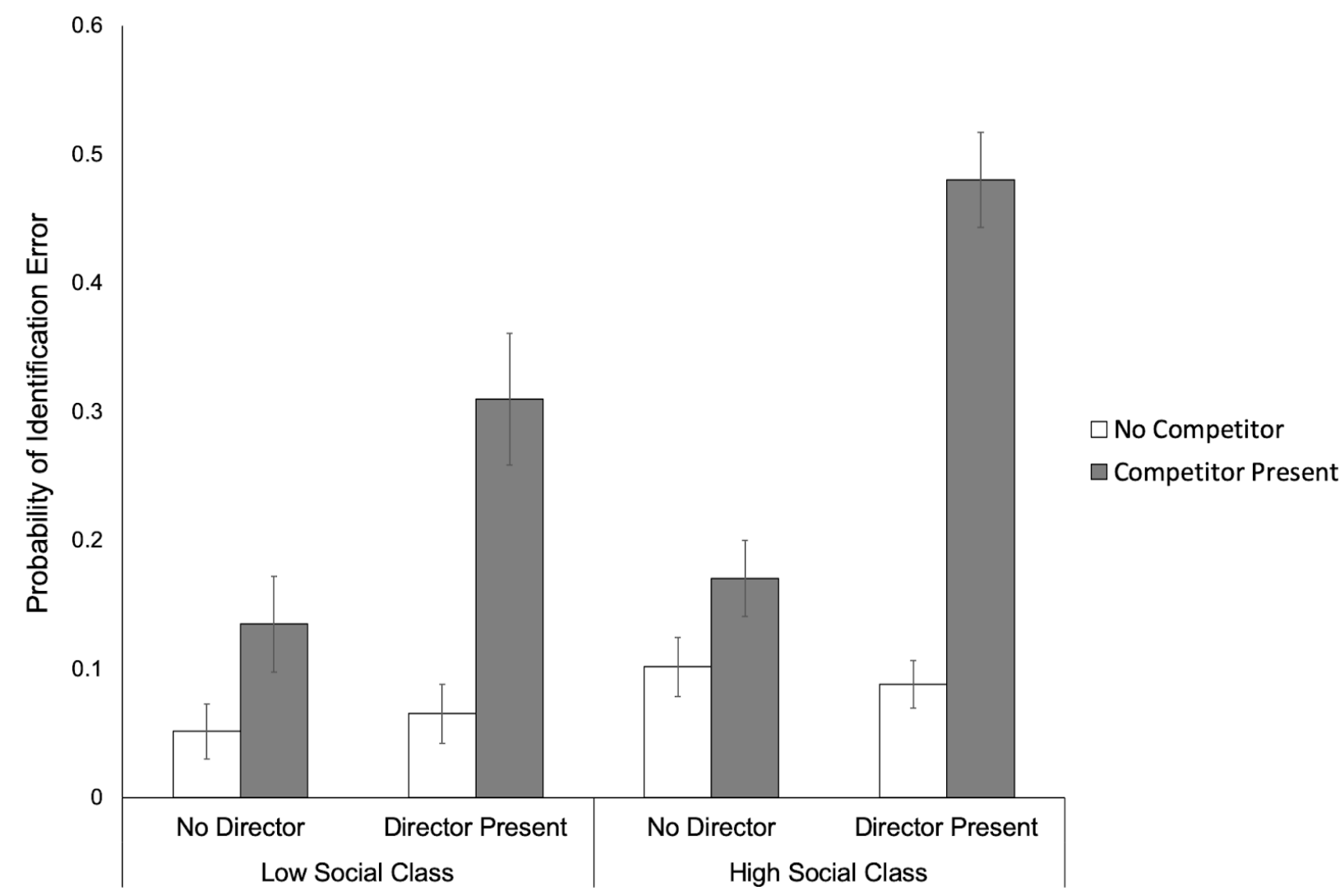

Figure 5. Object-identification error probability as a function of participant social class, the presence of a human director, and the presence of a competitor object (combined Studies 2A and 2B data). Error bars represent $95 \%$ confidence intervals.

\section{Robustness Against Confounds}

Any association between social class and other participant demographics could undermine our argument for the existence of class-based perspective-taking effects. Indeed, we find that social class is confounded with participants' gender and race; across studies, women tended to be lower and Asian participants tended to be higher in social class than their male and European American counterparts (Supplemental Table S3). In light of the association between social class, gender, and race, we conducted a further mixed-effects logistic regression — this time adding dummy-coded gender and race vectors and their interactions with director and 
competitor as predictors of object-identification errors. Because age is known to predict ToM performance (Dumontheil et al., 2010; Kirkland et al., 2013) age was also added as a covariate in the model. Inclusion of these covariates did not substantially alter the critical Social Class Category $\times$ Director $\times$ Competitor interaction $(\mathrm{B}=0.232, \mathrm{SE} \mathrm{B}=0.092, z=2.515, p=.012$, 95\% CI[0.051, 0.412]; Supplemental Table S4). ${ }^{2}$

\section{Discussion}

The results of two studies, one initial investigation and one preregistered replication, confirm our hypothesis that members of lower social classes outperform members of higher classes on a task measuring visual perspective-taking. The tendency of higher-class individuals to make more errors during the Director Task represents a failure to execute a fully-developed capacity, not a lack of ability. Thus, for the first time, we document that social class, like other cultural dimensions (e.g., national cultures), can affect the spontaneous deployment of a universal ToM competency.

\section{General Discussion}

The present studies suggest that higher social class is associated with attenuated Theory of Mind performance. First, we replicated the previously-observed negative association between social class and emotion perception (Kraus et al., 2010). Second, we provide evidence for a novel association between social class and perspective-taking: Higher-class individuals are worse than their lower-class counterparts at taking the visual perspective of another person. In order to

${ }^{2}$ Replicating Wu and Keysar (2007), and bolstering the construct validity of the Director Task, Asian participants displayed fewer perspective-taking errors than did European-American participants, as indicated by a significant negative Asian $\times$ Director $\times$ Competitor interaction (Supplemental Table S4). 
address criticisms of previous findings on class and emotion perception (Deveney et al., 2018), we employed a consistent measure of class (Dietze \& Knowles, 2016; Jackman \& Jackman, 1983) and an established emotion perception task (the Reading the Mind in the Eyes Test; Baron-Cohen et al., 2001). Likewise, our test of the relationship between visual perspective-taking benefited from the use of an established task (the Director Task; Dumontheil et al., 2010). Taken together, the current studies provide the most comprehensive methodological assessment of the relationship between ToM ability and social class to date.

\section{Inconsistent Findings Regarding Social Class and Emotion Perception}

Given the present findings, one might ask why Deveney and colleagues (2018) failed to reliably observe a negative relationship between social class and emotion perception. One reason, we argue, is that Deveney et al.'s (2018) studies utilized problematic samples. Specifically, three out of four samples are derived from the cognitive-testing website testmybrain.com. Given the nature of this site, the authors' conclusions may be limited by a reliance on self-selected groups of participants highly curious about psychology and self-knowledge. Additionally, one of the authors' studies (Study 2) used a nonstandard version of the RMET that did not provide participants with definitions of the relevant emotion terms - raising the possibility that class-based differences in education or vocabulary obscured a negative relationship between class and RMET scores. Two other studies (Studies 3 and 4) used tests that involved only basic emotions - and are thus unlikely to tap cultural differences in perceptual expertise. Indeed, the creators of the RMET describe it as an "advanced" test of ToM that should only yield population differences in terms of complex, and not basic, emotions (Baron-Cohen et al., 1997, 2001). Therefore, it is perhaps unsurprising that the only of Deveney 
and colleagues' (2018) studies to use the validated RMET (Study 1) succeeded in replicating an negative relationship between class and emotion perception. These considerations, coupled with the results of the present study, lead us to regard the evidence for an inverse association between social class and emotion perception as robust.

\section{Mechanisms Linking Social Class to Emotion Perception and Perspective Taking}

We have argued that social class impacts Theory of Mind competencies via class-based differences in motivational-relevance appraisals (see also Dietze \& Knowles, 2016). That is, lower-class individuals - owing to their greater levels of cultural interdependence - may appraise other human beings as more relevant to their goals and well-being than do higher-class individuals. Such appraisals are theorized to represent cultural "defaults" that affect social cognition pervasively and spontaneously. The present studies were not designed to test potential mechanisms linking relevance appraisals to class-based differences in emotion-perception and visual perspective-taking performance. Given differences in the nature of emotion-perception and perspective-taking failures, however, we can venture educated guesses concerning these mechanisms.

\section{Emotion Perception}

According to Baron-Cohen and colleagues (2001), correct responses on the RMET require test-takers to (a) "have a mental state lexicon and know the semantics of these terms" and (b) "match the eyes in each picture to examples of eye-region expressions stored in memory and seen in the context of particular mental states" (p. 241). A perfect score on the RMET thus entails knowing all the emotion labels in the test and possessing an adequately precise visual representation of each. In our view, it is unlikely that members of higher social classes possess a 
more limited emotional vocabulary than their lower-class counterparts. The present results therefore suggest that lower-class perceivers tend to possess more numerous and/or precise visual representations of complex emotions - that is, greater "perceptual expertise" (Carey, 1996) — than do higher-class perceivers.

Perceptual expertise in any domain is developed through practice. Effective practice involves making classifications and receiving feedback about the accuracy of one's attempts (Tanaka \& Gauthier, 1997). Sustained practice, in turn, depends at least in part on the personal importance that perceivers associate with, and thus the amount of attention they allocate to, the domain in question. We have argued here and elsewhere (Dietze \& Knowles, 2016) that lower-class perceivers are more likely than their higher-class counterparts to appraise other humans as motivationally relevant (i.e., important to their current goals and well-being). If we are correct in this, then lower-class adults' heightened emotion-recognition expertise may reflect a longstanding willingness to attend closely to others' facial expressions and attempt to infer the emotions they convey.

\section{Visual Perspective-Taking}

If errors of emotion recognition reflect a lack of ability, then errors of visual perspective-taking reflect the failure to appropriately execute a fully-developed capacity. In the Director Task, participants in the director condition are explicitly told that they should take his perspective into account when deciding which object to move. Thus, errors in the task occur either when participants forget that the director is present—or remember that he is present but fail to "select" his perspective instead of their own. Because participants are told to attend to the director, and because it is the director who supposedly issues the voice directives that begin 
every trial of the task, we regard the latter possibility as more likely. That is, rather than forgetting his presence entirely, higher-class participants may forget to calculate his perspective before rendering their responses in the task.

We suggest that lower-class perceivers, owing to their tendency to spontaneously appraise other people as motivationally relevant, are also more likely than higher-class perceivers to spontaneously calculate other people's perspectives. We see two potential mechanisms linking chronic relevance appraisals to the spontaneous computation of others' perspectives. First, it may be that lower-class individuals, because they regard others as relevant, become highly practiced at selecting their perspectives over their own. Such practice may subserve a process of "goal automatization" (Glaser \& Banaji, 1999; Knowles et al., 2001), in which the goal of calculating others' perspectives comes to be spontaneously triggered in others' mere presence. Alternatively, the goal of incorporating others' perspectives may form part of lower-class perceivers' representations of the "generic other" in much the same way that a range of relational goals form part of people's representations of significant others (Fitzsimons \& Bargh, 2003). Just as specific relational goals can be automatically activated in the presence of people's significant others (e.g., friends and family members), perspective-taking goals may, for lower-class perceivers, be automatically triggered in the presence of any others. In this case, lower-class perceivers' heightened motivational relevance appraisals result, not necessarily in the routinization of perspective-taking goals through practice, but in the embedding and automatic activation of such goals in representations of generic others. 


\section{Lower-Class Deficits — or Heightened Social Attunement?}

Implications for past and future research are far-reaching. Adults' performance on ToM tasks relies heavily on memory and executive function (Apperly et al., 2008). The fact that lower-class individuals outperform higher-class individuals across measures of ToM stands in clear contrast to the common discourse concerning neurocognitive deficits among the lower classes (for a review, see Hackman \& Farah, 2009). For example, decades of research document that lower-class individuals are more likely to exhibit attentional deficits and display impaired working memory performance. The evidence provided here suggests that these deficits may be domain-specific. Indeed, we show that lower-class performance outcomes are reversed in the social realm.

In addition, some of the evidence for deficits might itself be reinterpreted as artifacts of lower-class individuals' heightened social attunement and ToM competency. For example, Stevens and colleagues (2009) examined the effects of social class on auditory attention. Children listened to two stories simultaneously, one in each ear, and were asked to selectively attend to one story while event-related potentials (ERPs) were recorded. Although no class differences emerged in ERPs to the attended story, lower-class children exhibited a higher-amplitude response to the unattended channel. The authors interpret this pattern as reflecting lower-class children's difficulty in suppressing attention to distracting stimuli-that is, to a failure of executive function.

We believe another interpretation is possible. Specifically, given that no class differences emerged in memory for the attended channel, it may be that lower-class children were simply better at remaining attuned to social cues (i.e., the narration presented to the unattended ear) 
while still effectively encoding attended information. Thus, we see Stevens and colleagues (2009) as having measured, not lower-class failures of executive function, but rather reflexive attention to socially-relevant speech. Such information, of course, is saturated with cues to others' intentions, desires, and beliefs—and thus closely resembles a ToM competency. Low social class may thus be associated with a heightened ability to "socially multitask" - that is, to maintain attention as instructed while still monitoring other sources of socially-relevant information. We stress that this interpretation does not explain the experiences of lower-class people who experience chronic poverty, stress, or malnutrition; the severity of these circumstances can be a detriment to psychological functioning and development, including in the social domain.

\section{Limitations and Future Directions}

We note several limitations of the current research. It was necessary to administer the Director Task in the lab and thus, in Study 2A and 2B, we were limited to a convenience sample comprised of college undergraduates. Future research should attempt to replicate the results in other populations. Furthermore, we choose the Director Task and the Reading the Mind in the Eyes Test because they are well-validated ToM tasks that capture two different facets of ToM. However, ToM includes additional facets (and tasks) that, in association with social class, provide an interesting avenue for further investigation. For example, false-belief tasks for adults, in which individuals ignore privileged knowledge and overcome egocentric biases could be more difficult for higher-class individuals, who tend to exhibit greater self-focus than their lower-class counterparts. In addition, eye-tracking and fMRI paradigms have shown that false-belief tasks involve implicit belief processing (Schneider et al., 2012, 2014); thus, future research employing 
similar techniques could potentially shed light on the mechanisms linking social class to ToM performance. 


\section{Author Contributions}

P. Dietze and E. D. Knowles developed the study, designed the experiments, collected and analyzed the data, and wrote the manuscript. 


\section{References}

Adler, N. E., Epel, E. S., Castellazzo, G., \& Ickovics, J. R. (2000). Relationship of subjective and objective social status with psychological and physiological functioning: Preliminary data in healthy, White women. Health psychology, 19, 586.

Apperly, I. A. (2012). What is "theory of mind"? Concepts, cognitive processes and individual differences. Quarterly Journal of Experimental Psychology, 65, 825-839.

Apperly, I. A., Back, E., Samson, D., \& France, L. (2008). The cost of thinking about false beliefs: evidence from adults' performance on a non-inferential theory of mind task. Cognition, 106, 1093-1108.

Arterberry, M. E., Bornstein, M. H., \& Blumenstyk, J. B. (2013). Categorization of two-dimensional and three-dimensional stimuli by 18-month-old infants. Infant Behavior \& Development, 36, 786-795.

Astington, J. W., Harris, P. L., \& Olson, D. R. (1988). Developing Theories of Mind. Cambridge University Press.

Balliet, D., Li, N. P., Macfarlan, S. J., \& Van Vugt, M. (2011). Sex differences in cooperation: a meta-analytic review of social dilemmas. Psychological Bulletin, 137, 881-909.

Baron-Cohen, S., Jolliffe, T., Mortimore, C., \& Robertson, M. (1997). Another advanced test of theory of mind: Evidence from very high functioning adults with autism or Asperger syndrome. Journal of Child Psychology and Psychiatry, and Allied Disciplines, 38, $813-822$.

Baron-Cohen, S., Wheelwright, S., Hill, J., Raste, Y., \& Plumb, I. (2001). The "Reading the 
Mind in the Eyes" Test revised version: a study with normal adults, and adults with Asperger syndrome or high-functioning autism. Journal of Child Psychology and Psychiatry, and Allied Disciplines, 42, 241-251.

Bourdieu, P. (1986). The Forms of Capital. In J. G. Richardson (Ed.), Handbook of theory and research for the sociology of education (pp. 241-258). Greenwood Press.

Buhrmester, M., Kwang, T., \& Gosling, S. D. (2011). Amazon's Mechanical Turk: A New Source of Inexpensive, Yet High-Quality, Data? Perspectives on Psychological Science: A Journal of the Association for Psychological Science, 6, 3-5.

Call, J., \& Tomasello, M. (2008). Does the chimpanzee have a theory of mind? 30 years later. Trends in Cognitive Sciences, 12, 187-192.

Carey, S. (1996). Perceptual classification and expertise. Perceptual and Cognitive Development, $49-69$.

Curran, P. J., \& Hussong, A. M. (2009). Integrative data analysis: the simultaneous analysis of multiple data sets. Psychological Methods, 14, 81-100.

Darmon, N., \& Drewnowski, A. (2008). Does social class predict diet quality? The American Journal of Clinical Nutrition, 87, 1107-1117.

Deveney, C. M., Chen, S. H., Wilmer, J. B., Zhao, V., Schmidt, H. B., \& Germine, L. (2018). How generalizable is the inverse relationship between social class and emotion perception? PloS One, 13, e0205949.

Dietze, P., \& Knowles, E. D. (2016). Social Class and the Motivational Relevance of Other Human Beings: Evidence From Visual Attention. Psychological Science, 27, 1517-1527. Dumontheil, I., Apperly, I. A., \& Blakemore, S.-J. (2010). Online usage of theory of mind 
continues to develop in late adolescence. Developmental Science, 13, 331-338.

Eagly, A. H. (2009). The his and hers of prosocial behavior: an examination of the social psychology of gender. The American Psychologist, 64, 644-658.

Eisenberg, N., Murphy, B. C., \& Shepard, S. (1997). The development of empathic accuracy. In W. Ickes (Ed.), Empathic accuracy (pp. 73-116). Guilford Press.

Fitzsimons, G. M., \& Bargh, J. A. (2003). Thinking of you: nonconscious pursuit of interpersonal goals associated with relationship partners. Journal of Personality and Social Psychology, 84, 148-164.

Flavell, J. H., Everett, B. A., Croft, K., \& Flavell, E. R. (1981). Young children's knowledge about visual perception: Further evidence for the Level 1-Level 2 distinction. Developmental Psychology, 17, 99-103.

Glaser, J., \& Banaji, M. R. (1999). When fair is foul and foul is fair: reverse priming in automatic evaluation. Journal of Personality and Social Psychology, 77, 669-687.

Grossmann, I., \& Varnum, M. E. W. (2011). Social class, culture, and cognition. Social Psychological and Personality Science, 2, 81-89.

Hackman, D. A., \& Farah, M. J. (2009). Socioeconomic status and the developing brain. Trends in Cognitive Sciences, 13, 65-73.

Jackman, M. R., \& Jackman, R. W. (1983). Class Awareness in the United States. Univ of California Press.

Keysar, B. (1997). Unconfounding common ground. Discourse Processes, 24, 253-270.

Keysar, B., Lin, S., \& Barr, D. J. (2003). Limits on theory of mind use in adults. Cognition, 89, $25-41$. 
Kirkland, R. A., Peterson, E., Baker, C. A., Miller, S., \& Pulos, S. (2013). Meta-analysis Reveals Adult Female Superiority in" Reading the Mind in the Eyes Test." North American Journal of Psychology, 15.

Knowles, E. D., Morris, M. W., Chiu, C.-Y., \& Hong, Y.-Y. (2001). Culture and the Process of Person Perception: Evidence for Automaticity among East Asians in Correcting for Situational Influences on Behavior. Personality \& Social Psychology Bulletin, 27, $1344-1356$.

Kraus, M. W., Côté, S., \& Keltner, D. (2010). Social class, contextualism, and empathic accuracy. Psychological Science, 21, 1716-1723.

Kraus, M. W., \& Keltner, D. (2009). Signs of socioeconomic status: a thin-slicing approach. Psychological Science, 20, 99-106.

Kraus, M. W., Piff, P. K., Mendoza-Denton, R., Rheinschmidt, M. L., \& Keltner, D. (2012). Social class, solipsism, and contextualism: how the rich are different from the poor. Psychological Review, 119, 546-572.

Lachman, M. E., \& Weaver, S. L. (1998). The sense of control as a moderator of social class differences in health and well-being. Journal of Personality and Social Psychology, 74, $763-773$.

Luk, K. K. S., Xiao, W. S., \& Cheung, H. (2012). Cultural effect on perspective taking in Chinese-English bilinguals. Cognition, 124, 350-355.

Malle, B. F., Moses, L. J., \& Baldwin, D. A. (2001). Intentions and Intentionality: Foundations of Social Cognition. MIT Press.

Markus, H. R., \& Kitayama, S. (1991). Culture and the self: Implications for cognition, emotion, 
and motivation. Psychological Review, 98, 224-253.

Moll, H., \& Tomasello, M. (2006). Level 1 perspective-taking at 24 months of age. British Journal of Developmental Psychology, 24, 603-613.

Montagne, B., Kessels, R. P. C., Frigerio, E., de Haan, E. H. F., \& Perrett, D. I. (2005). Sex differences in the perception of affective facial expressions: do men really lack emotional sensitivity? Cognitive Processing, 6, 136-141.

Peer, E., Samat, S., Brandimarte, L., \& Acquisti, A. (2017). Beyond the Turk: An Empirical Comparison of Alternative Platforms for Online Behavioral Research. Journal of Experimental Social Psychology, 153-163.

Piff, P. K., Stancato, D. M., Côté, S., Mendoza-Denton, R., \& Keltner, D. (2012). Higher social class predicts increased unethical behavior. Proceedings of the National Academy of Sciences of the United States of America, 109, 4086-4091.

Piff, P. K., Stancato, D. M., Martinez, A. G., Kraus, M. W., \& Keltner, D. (2012). Class, chaos, and the construction of community. Journal of Personality and Social Psychology, 103, 949-962.

Schneider, D., Bayliss, A. P., Becker, S. I., \& Dux, P. E. (2012). Eye movements reveal sustained implicit processing of others' mental states. Journal of Experimental Psychology. General, 141, 433-438.

Schneider, D., Slaughter, V. P., Becker, S. I., \& Dux, P. E. (2014). Implicit false-belief processing in the human brain. NeuroImage, 101, 268-275.

Shahaeian, A., Peterson, C. C., Slaughter, V., \& Wellman, H. M. (2011). Culture and the sequence of steps in theory of mind development. Developmental Psychology, 47, 
$1239-1247$.

Slaughter, V., \& Perez-Zapata, D. (2014). Cultural Variations in the Development of Mind Reading. Child Development Perspectives, 8, 237-241.

Sodian, B., Thoermer, C., \& Metz, U. (2007). Now I see it but you don't: 14-month-olds can represent another person's visual perspective. Developmental Science, 10, 199-204.

Sprong, M., Schothorst, P., Vos, E., Hox, J., \& Van Engeland, H. (2007). Theory of mind in schizophrenia. British Journal of Psychiatry, 191, 5-13.

Stellar, J. E., Manzo, V. M., Kraus, M. W., \& Keltner, D. (2012). Class and compassion: Socioeconomic factors predict responses to suffering. Emotion, 12, 449-459.

Stephens, N. M., Fryberg, S. A., Markus, H. R., Johnson, C. S., \& Covarrubias, R. (2012). Unseen disadvantage: how American universities' focus on independence undermines the academic performance of first-generation college students. Journal of Personality and Social Psychology, 102, 1178-1197.

Stephens, N. M., Markus, H. R., \& Phillips, L. T. (2014). Social class culture cycles: how three gateway contexts shape selves and fuel inequality. Annual Review of Psychology, 65, $611-634$.

Stevens, C., Lauinger, B., \& Neville, H. (2009). Differences in the neural mechanisms of selective attention in children from different socioeconomic backgrounds: an event-related brain potential study. Developmental Science, 12, 634-646.

Tanaka, J. W., \& Gauthier, I. (1997). Expertise in object and face recognition. Psychology of Learning and Motivation, 36, 83-125.

Wellman, H. M. (2014). Making minds: How theory of mind develops. Oxford University Press. 
Wellman, H. M., Cross, D., \& Watson, J. (2001). Meta-analysis of theory-of-mind development: The truth about false belief. Child Development, 72, 655-684.

Wellman, H. M., Fang, F., Liu, D., Zhu, L., \& Liu, G. (2006). Scaling of theory-of-mind understandings in Chinese children. Psychological Science, 17, 1075-1081.

Wu, S., \& Keysar, B. (2007). The effect of culture on perspective taking. Psychological Science, $18,600-606$.

Yirmiya, N., Erel, O., Shaked, M., \& Solomonica-Levi, D. (1998). Meta-analyses comparing theory of mind abilities of individuals with autism, individuals with mental retardation, and normally developing individuals. Psychological Bulletin, 124, 283-307. 


\section{Supplementary Online Materials}

"Social Class Predicts Emotion Perception and Perspective-Taking Performance in Adults" (Dietze \& Knowles)

\section{Materials and Procedure for Study 1}

In both studies (Study 1A and 1B), we recorded the total time it took participants to complete the study. In both studies, the full questionnaire assessed agreeableness (Rammstedt \& John, 2012), gender, age, ethnicity, and political ideology. Participants also answered questions concerning their own income, their household income, their household income while growing up, their own and their parents' educational attainment, the subjective SES ladder (Adler, Epel, Castellazzo, \& Ickovics, 2000), sense of power (Anderson \& Galinsky, 2006), and scale measures of their current and childhood SES (Mittal \& Griskevicius, 2014). The complete data set and analysis code can be found in the Open Science Framework online repository:

\section{https://osf.io/z9xta/.}

\section{Results for Study 1}

\section{Robustness Against Confounds (combined Studies $1 A$ and 1B)}

Table S1. Regression of social class on other participant demographics (combined Studies 1A and 1B).

\begin{tabular}{l|cccccc}
\hline \multirow{1}{*}{ Predictor } & \multicolumn{1}{c}{} & & & & \multicolumn{2}{c}{$95 \% \mathrm{Cl}$} \\
Age & $\mathrm{B}$ & SE B & $t$ & $p$ & LB & UB \\
Female & $7.16 \times 10^{-4}$ & 0.003 & 0.255 & 0.799 & -0.005 & 0.006 \\
Black & -0.058 & 0.032 & -1.851 & 0.065 & -0.120 & 0.004 \\
Latino & -0.302 & 0.114 & -2.644 & 0.008 & -0.526 & -0.078 \\
Asian & -0.173 & 0.130 & -1.333 & 0.183 & -0.427 & 0.082 \\
Other race & 0.041 & 0.137 & 0.300 & 0.765 & -0.228 & 0.311 \\
\hline
\end{tabular}


Note. Racial categories dummy coded with White as the reference category. Gender coded such that $-1=$ male and $1=$ female.

Table S2. Regression of RMET scores on social class category and other participant demographics (combined Studies $1 \mathrm{~A}$ and $1 \mathrm{~B}$ data).

\begin{tabular}{l|cccccc}
\hline \multirow{2}{*}{\multicolumn{1}{c|}{ Predictor }} & \multicolumn{1}{c}{} & & \multicolumn{2}{c}{$95 \% \mathrm{Cl}$} \\
\hline Social Class Category & $\mathbf{- 1 . 2 9 6}$ & $\mathbf{0 . 1 9 7}$ & $-\mathbf{6 . 2 6 8}$ & $\mathbf{1 . 0 0 2 \times 1 0 ^ { - 1 0 }}$ & $\mathbf{- 1 . 6 8 3}$ & $\mathbf{- 0 . 9 0 8}$ \\
Age & 0.021 & 0.015 & 1.405 & 0.161 & -0.008 & 0.050 \\
Female & 0.708 & 0.166 & 4.262 & $2.288 \times 10^{-5}$ & 0.382 & 1.034 \\
Black & -1.968 & 0.616 & -3.240 & 0.001 & -3.177 & -0.759 \\
Latino & 0.423 & 0.682 & 0.952 & 0.535 & -0.916 & 1.763 \\
Asian & -0.899 & 0.738 & -0.883 & 0.223 & -2.348 & 0.549 \\
Other race & -2.811 & 0.795 & -2.258 & $4.293 \times 10^{-4}$ & -4.372 & -1.251 \\
\hline
\end{tabular}

Note. Racial categories dummy coded with White as the reference category. Gender coded such that $-1=$ male and $1=$ female.

\section{Materials and Procedure for Study 2}

In Study 2A and Study 2B, the full questionnaire assessed participants gender, age, ethnicity, and political ideology. Participants also answered questions concerning their own income, their current caretakers' household income, their caretakers' household income while growing up, their own and their parents' educational attainment, the subjective SES ladder (Adler, Epel, Castellazzo, \& Ickovics, 2000), and scale measures of their current and childhood SES (Mittal \& Griskevicius, 2014). The complete data set and analysis code can be found in the Open Science Framework online repository: https://osf.io/jxbsm/.

\section{Results for Study 2}




\section{Robustness Against Confounds (combined Studies $2 A$ and $2 B$ )}

Table S3. Regression of social class on other participant demographics (combined Studies $2 \mathrm{~A}$ and 2B data).

\begin{tabular}{|c|c|c|c|c|c|c|}
\hline \multirow[b]{2}{*}{ Predictor } & \multirow[b]{2}{*}{ B } & \multirow[b]{2}{*}{ SE B } & \multirow[b]{2}{*}{$t$} & \multirow[b]{2}{*}{$p$} & \multicolumn{2}{|c|}{$95 \% \mathrm{Cl}$} \\
\hline & & & & & LB & UB \\
\hline Age & -0.701 & 0.297 & -2.365 & 0.020 & -1.289 & -0.114 \\
\hline Female & -0.744 & 0.251 & -2.963 & 0.004 & -1.241 & -0.247 \\
\hline Black & -0.269 & 0.199 & -1.351 & 0.179 & -0.664 & 0.125 \\
\hline Latino & -0.157 & 0.295 & -0.533 & 0.595 & -0.741 & 0.426 \\
\hline Asian & 0.117 & 0.064 & 1.814 & 0.072 & -0.011 & 0.245 \\
\hline Other race & 0.029 & 0.084 & 0.347 & 0.730 & -0.137 & 0.195 \\
\hline
\end{tabular}

Note. Racial categories dummy coded with White as the reference category. Gender coded such that $-1=$ male and $1=$ female.

Table S4. Mixed-effects logistic regression analysis of identification errors in the Director Task with covariates (combined Studies 2A and 2B data).

\begin{tabular}{|c|c|c|c|c|c|c|}
\hline \multirow[b]{2}{*}{ Predictor } & \multirow[b]{2}{*}{ B } & \multirow[b]{2}{*}{ SE B } & \multirow[b]{2}{*}{$z$} & \multirow[b]{2}{*}{$p$} & \multicolumn{2}{|c|}{$95 \% \mathrm{Cl}$} \\
\hline & & & & & LB & UB \\
\hline Social Class Category (SCC) & 0.596 & 0.263 & 2.270 & 0.023 & 0.081 & 1.111 \\
\hline Director (D) & 0.781 & 0.247 & 3.165 & 0.002 & 0.297 & 1.264 \\
\hline Competitor (C) & 1.299 & 0.313 & 4.154 & $3.267 \mathrm{e}-05$ & 0.686 & 1.912 \\
\hline$S C C \times D$ & -0.019 & 0.150 & -0.125 & 0.900 & -0.313 & 0.275 \\
\hline $\mathrm{SCC} \times \mathrm{C}$ & 0.148 & 0.191 & 0.775 & 0.438 & -0.226 & 0.522 \\
\hline$D \times C$ & 0.479 & 0.148 & 3.226 & 0.001 & 0.188 & 0.770 \\
\hline$S C C \times D \times C$ & 0.232 & 0.092 & 2.515 & 0.012 & 0.051 & 0.412 \\
\hline Age & -0.852 & 0.245 & -3.469 & $5.227 e-04$ & -1.333 & -0.370 \\
\hline Age $\times D$ & 0.381 & 0.148 & 2.582 & 0.010 & 0.092 & 0.671 \\
\hline Age $\times C$ & -0.239 & 0.177 & -1.352 & 0.176 & -0.586 & 0.108 \\
\hline Age $\times D \times C$ & 0.111 & 0.091 & 1.219 & 0.223 & -0.067 & 0.290 \\
\hline Female & 0.049 & 0.241 & 0.204 & 0.839 & -0.423 & 0.522 \\
\hline Female $\times D$ & 0.264 & 0.135 & 1.957 & 0.050 & $-4.35 e-04$ & 0.529 \\
\hline Female $\times C$ & -0.041 & 0.174 & -0.237 & 0.812 & -0.383 & 0.300 \\
\hline Female $\times \mathrm{D} \times \mathrm{C}$ & 0.161 & 0.082 & 1.971 & 0.049 & $8.78 \mathrm{e}-04$ & 0.321 \\
\hline Black & -0.041 & 0.875 & -0.047 & 0.963 & -1.756 & 1.674 \\
\hline Black $\times D$ & 0.452 & 0.509 & 0.887 & 0.375 & -0.546 & 1.450 \\
\hline
\end{tabular}




\begin{tabular}{|c|c|c|c|c|c|c|}
\hline Black $\times \mathrm{C}$ & 0.006 & 0.638 & 0.009 & 0.993 & -1.245 & 1.256 \\
\hline Black $\times D \times C$ & 0.326 & 0.308 & 1.059 & 0.290 & -0.277 & 0.928 \\
\hline Latino & -0.637 & 0.752 & -0.847 & 0.397 & -2.111 & 0.837 \\
\hline Latino $\times \mathrm{D}$ & 0.571 & 0.415 & 1.375 & 0.169 & -0.243 & 1.385 \\
\hline Latino $\times \mathrm{C}$ & 0.364 & 0.536 & 0.678 & 0.498 & -0.687 & 1.414 \\
\hline Latino $\times \mathrm{D} \times \mathrm{C}$ & 0.049 & 0.241 & 0.204 & 0.838 & -0.423 & 0.521 \\
\hline Asian & -0.687 & 0.575 & -1.194 & 0.232 & -1.815 & 0.441 \\
\hline Asian $\times D$ & 0.497 & 0.326 & 1.523 & 0.128 & -0.142 & 1.136 \\
\hline Asian $\times C$ & 0.028 & 0.413 & 0.069 & 0.945 & -0.782 & 0.838 \\
\hline Asian $\times D \times C$ & -0.456 & 0.189 & -2.409 & 0.016 & -0.828 & -0.085 \\
\hline Other Race & -0.025 & 0.819 & -0.031 & 0.975 & -1.630 & 1.579 \\
\hline Other Race $\times \mathrm{D}$ & 0.038 & 0.440 & 0.086 & 0.931 & -0.825 & 0.901 \\
\hline Other Race $\times C$ & 0.061 & 0.577 & 0.105 & 0.916 & -1.070 & 1.192 \\
\hline Other Race $\times D \times C$ & -0.174 & 0.247 & -0.705 & 0.481 & -0.659 & 0.310 \\
\hline
\end{tabular}

Note. Director and competitor coded such that $-1=$ no director or competitor object and $1=$ director or competitor object present. Social class centered at "middle class." Social class category centered at "middle class." The SSC $\times \mathrm{D} \times \mathrm{C}$ interaction reflects more perspective-taking failures among higher-class participants than among lower-class participants, adjusting for other participant demographics. 


\section{References}

Adler, N. E., Epel, E. S., Castellazzo, G., \& Ickovics, J. R. (2000). Relationship of subjective and objective social status with psychological and physiological functioning: preliminary data in healthy white women. Health Psychology: Official Journal of the Division of Health Psychology, American Psychological Association, 19(6), 586-592.

Anderson, C., \& Galinsky, A. D. (2006). Power, optimism, and risk-taking. European Journal of Social Psychology. https://doi.org/10.1002/ejsp.324

Mittal, C., \& Griskevicius, V. (2014). Sense of control under uncertainty depends on people's childhood environment: a life history theory approach. Journal of Personality and Social Psychology, 107(4), 621-637.

Rammstedt, B., \& John, O. P. (2012). Big Five Inventory-10. PsycTESTS Dataset. https://doi.org/10.1037/t01744-000 